\title{
Espacio, lugar y tiempo: la reapropiación social de la naturaleza y la construcción local de la racionalidad ambiental
}

\author{
Enrique LEFF*
}

\begin{abstract}
RESUMO
Diversidade cultural e preservação das identidades dos povos são fundamentais para viabilizar o desenvolvimento sustentável em escala local e global. Estilos étnicos organizam as práticas de uso da natureza e das populações indígenas e das sociedades camponesas. Assim, a cultura aparece revalorizada como um recurso para o desenvolvimento sustentável. O presente artigo pretende repensar o espaço e o tempo, através de uma racionalidade ambiental que ressignifique a natureza e a cultura, como uma alternativa ao logocentrismo da racionalidade científica e a globalização homogeneizante dominantes.
\end{abstract}

Palavras-chave: Racionalidade Ambiental, Cultura, Sustentabilidade, Identidade

\section{RESUMEN}

La diversidad cultural y la preservación de las identidades de los pueblos son fundamentales para viabilizar el desarrollo sustentable a escala local y global. Los estilos étnicos organizan las prácticas de uso de la naturaleza de las poblaciones indígenas y de las sociedades campesinas. Así, la cultura aparece revalorizada como un recurso para el desarrollo sustentable. El propósito del presente artículo es repensar el espacio y el tiempo a través de una racionalidad ambiental que resignifique a la naturaleza y a la cultura, en oposición al logocentrismo de la racionalidad científica y a la globalización homogeneizante dominantes.

Palavras-chave: Racionalidad Ambiental, Cultura, Sustentabilidad, Identidad

\begin{abstract}
Cultural diversity and the preservation of peoples identities are fundamental for making sustainable development at local and global levels viable. The practices of nature use by indigenous people and peasant societies are organized by ethnic styles. Thus, culture acquires new value as a resource for sustainable development. The purpose of this article is to rethink space and time, and to resignify nature and culture in the perspective of an environmental rationality, as an alternative to logocentrism in scientific rationality and dominant homogeneizing globalization.

Key-words: Environmental rationality, Culture, Sustainability, Identity

* Coordinador de la Red de Formación Ambiental para América Latina y el Caribe, PNUMA.
\end{abstract}




\section{Cultura y sustentabilidad}

Toda organización cultural es un complejo sistema de valores, ideologías, significados, prácticas productivas y estilos de vida que se han desarrollado a lo largo de la historia y se especifican en diferentes contextos geográficos y ecológicos. En este sentido, los principios de una "cultura ecológica", que movilizan y guían los procesos sociales hacia el desarrollo sustentable, están arraigados en racionalidades culturales constituidas por las diferentes formas de organización simbólica y productiva de los pueblos indios y las comunidades campesinas. La degradación ambiental y la destrucción de sus recursos, causados por el proceso de crecimiento y globalización económica y enmascaradas hoy en día por el propósito de un "desarrollo sostenible", han estado asociadas a la desintegración de valores culturales, identidades y prácticas productivas de las "sociedades tradicionales". Frente a estos procesos dominantes, las estrategias alternativas para el desarrollo sustentable, basadas en la diversidad cultural, están legitimando los derechos de las comunidades sobre sus territorios y espacios étnicos, sobre sus costumbres e instituciones sociales, y por la autogestión de sus recursos productivos. Los principios de diversidad en el ambientalismo enfrentan la homogeneidad de patrones productivos, defendiendo los valores de la diversidad de contextos ecológicos, la pluralidad cultural y la preservación de las identidades de los pueblos. Estos principios éticos aparecen como una condición para alcanzar los objetivos del desarrollo sustentable a escala local y global (Leff, 1998).

Las condiciones ecológicas y culturales de la sustentabilidad se han incorporado en las prácticas productivas de las sociedades tradicionales, y se reflejan tanto en sus formaciones simbólicas como en sus instrumentos tecnológicos, configurados en largos procesos de coevolución con la naturaleza, de transformación ambiental y asimilación cultural (Levy-Strauss, 1972; Descola, 1996). Las prácticas productivas fundadas en la simbolización cultural del ambiente, en creencias re- ligiosas y en significados sociales asignados a la naturaleza, han generado diferentes formas de percepción y apropiación, reglas sociales de acceso y uso, prácticas de gestión de ecosistemas y patrones de producción y consumo de recursos. De esta manera se configuraron las "ideologías agrícolas tradicionales" (Alcorn, 1989) y se desarrollaron las "estrategias productivas mesoamericanas" (Boege, 1988), basadas en el uso múltiple y sustentable de los "ecosistemas-recurso" (Morello, 1986).

Para entender el funcionamiento de estas estrategias culturales en el manejo sustentable de recursos naturales, es necesario comprender la racionalidad cultural que subyace a las clasificaciones de la naturaleza -la taxonomía folk — que refleja el conocimiento local de diferentes grupos étnicos, es decir, los sistemas de creencias, saberes y prácticas que forman sus "modelos holísticos" de percepción y uso de los recursos (Pitt, 1985). Estas formas de significación están íntimamente relacionadas con identidades culturales que van configurando estilos étnicos (Leroi-Gourhan, 1965) que organizan prácticas de uso de la naturaleza, constituyendo un patrimonio de recursos naturales y culturales de las poblaciones indígenas y las sociedades campesinas.

En su encuentro con civilizaciones dominadoras en el curso de la historia, estas culturas autóctonas han resistido y asimilado procesos de aculturación y cambio tecnológico, reafirmando y transformando sus rasgos identitarios. Así, las culturas indígenas americanas han preservado y redefinido sus identidades en sus encuentros y fusiones interétnicas en el período prehispánico y en los procesos de mestizaje que siguieron después de la conquista española. De forma similar, la supervivencia de los "pueblos originarios" en nuestros días, frente a las estrategias económicas y tecnológicas para reapropiarse la naturaleza y la biodiversidad-, está llevando a las poblaciones indígenas a resignificar sus identidades y a hibridizar sus culturas (Escobar, 1997a, 1997b).

Hoy en día, la cultura está siendo revalorizada como un "recurso para el desarrollo sustentable". ${ }^{1}$ En esta perspectiva, el legado cultural de los indígenas en

1“La cultura es el complemento de los recursos naturales en los sistemas productivos campesino-indígenas; la cultura orienta el uso de los recursos, mientras que éstos condicionan, hasta cierto grado, las opciones de vida del grupo étnico. Así concebida, la cultura es un recurso social, capaz de usarse destructiva o racionalmente, de perderse o desarrollarse." (Varèse; Martin, 1993, p. 738). 
Latinoamérica aparece como una parte integral de su patrimonio de recursos naturales, definido a través de las relaciones simbólicas y productivas que han guiado la coevolución de naturaleza y la cultura a través del tiempo. La organización cultural de las etnias y de las sociedades campesinas tradicionales establece un sistema de relaciones sociales y ecológicas de producción que da soporte a las prácticas de manejo integrado y sustentable de los recursos naturales ${ }^{2}$.

Varios autores han estudiado el funcionamiento de la racionalidad ecológica de las prácticas productivas arraigadas el "estilo de desarrollo prehispánico" (Gligo y Morello, 1980), el "modo de producción campesino" (Toledo, 1980), las "complementariedades ecosimbióticas verticales" (Condarco y Murra, 1987) y la "utopía andina" (Burgoa y Flores Galindo, 1982). Las culturas prehispánicas habían internalizado las bases y las potencialidades ecológicas a sus formas de organización productiva para el uso sustentable de la tierra y de los recursos naturales. Estas prácticas tomaron en cuenta las complementariedades de la diversidad ecológica y los espacios geográficos, integrando regiones que se extendían más allá de los territorios de un grupo étnico particular. Esta estrategia permitió optimizar la oferta ecológica de diversas geografías, del uso estacional de diferentes de los espacios productivos y de la fuerza de trabajo, de los ciclos y pisos ecológicos, así como de la fertilidad de la tierra y los procesos de regeneración de los recursos, para ensayar diferentes estrategias de policultivos, integrando la producción local al espacio territorial a través del comercio interregional y el intercambio intercomunal de excedentes económicos.

Los espacios étnicos de la América Tropical fueron y continúan siendo escenarios de estrategias de supervivencia y de etno-eco-desarrollo mediante la adaptación y transformación del ambiente; allí se desarrollaron importantes tecnologías agrícolas y trabajos públicos para el uso sustentable de recursos hidrológicos y para el incremento de la fertilidad de la tierra a través de técnicas para la conservación de agua y la prevención de erosión, así como de variadas innovaciones y estrategias agroecológicas: terrazas, chinampas, andenes y camellones (Murra, 1975; Romanini, 1876; Denevan, 1980a, 1980b; Masuda et al., 1985; CEPAL/PNUMA, 1983; de la Torre y Burgoa, 1986; Uribe, 1988; San Martín Arzabe, 1990, Altieri y Nicholls, 2000). Este vasto repertorio de conocimientos técnicos y prácticas productivas permitió el desarrollo y guió la evolución de las diferentes culturas que habitan la región andina y las zonas tropicales americanas.

\section{Complementariedades ecológicas, temporalidades culturales e identidades étnicas.}

Las estrategias productivas desarrolladas por diferentes culturas indígenas americanas implicaron el desarrollo de prácticas específicas de uso de los recursos de cada grupo étnico. Esta "cultura ecológica" fue integrada en las relaciones sociales y las fuerzas productivas de las sociedades rurales "tradicionales". El ordenamiento ecológico de procesos productivos operó a través de la complementariedad de espacios territoriales y ciclos ecológicos para el manejo sustentable y productivo de recursos naturales: estaciones de lluvia y sequía; distribución anual de diferentes cosechas seleccionadas por patrones de crecimiento diferenciados y por las condiciones climáticas de cada estación; el uso integral de plantas específicas y el manejo integrado de variedades genéticas de diferentes especies (maíz, papas), dependiendo de las condiciones topográficas y la calidad del suelo; de diferentes estrategias de uso fi-

2 "El patrimonio cultural es un recurso importante para la región. El logro de la sustentabilidad en las grandes estrategias agrícolas de los Andes, en las selvas tropicales y en las tierras anegadizas, requerirá la incorporación de tecnologías mayas, incaicas y preincaicas, aztecas y de otras etnias. Tales etnias campesinas poseen un riquísimo patrimonio tecnológico, cuyo su deterioro ha provocado enormes costos ecológicos en numerosos países, especialmente en México y Perú. Ellos lograron resolver problemas en los que la tecnología del Norte ha fracasado [...] como en articular el policultivo agrícola en pequeños desmontes con el uso extensivo de la selva contigua (lo hacen los descendientes de los mayas); manejar rodeos mixtos multipropósito para sobrevivir en climas semiáridos de alta variabilidad (los aymaras y los quechua en Bolivia); manejar la selva caducifolia para trasformarla en ecosistema poliproductivo, incluso en épocas de sequías extraordinarias; desarrollar germoplasma que responda a climas de baja predectibilidad y de lluvias (variedades de maíz y frijol de ciclos muy cortos, cortos y largos); desarrollar variedades adaptables a distintos pisos altitudinales (incas, mayas) y al gradiente latitudinal (pueblos andinos, mayas)... (Morello, 1990). 
nal (autoconsumo/mercado) e insumos tecnológicos (maquinaria, fertilizantes). Estas estrategias de diversificación y complementariedad de funciones ecológicas generaron diferentes prácticas de cultivos múltiples y combinados, así como de uso integrado de los recursos naturales en huertos familiares, milpas y acahuales; éstas significaron importantes estrategias de aprovechamiento sustentable del bosque tropical, mediante el manejo de la sucesión secundaria de los ecosistemas y procesos de regeneración selectiva de los recursos.

Estos "estilos prehispánicos de desarrollo sustentable", caracterizados por la articulación productiva de diferentes ecosistemas y territorios étnicos, provenían de la percepción de la naturaleza como un proceso sinergético e integrado y no como un acervo (stock) de recursos discretos. Estas estrategias optimizaron el uso del trabajo a través de la diversificación de la producción, ajustándose a las condiciones ecológicas y al potencial ambiental de cada región, integrando actividades agroforestales con la caza, la pesca y la recolección de especies vegetales. Este estilo de desarrollo fomentó la complementariedad de los procesos de trabajo y las prácticas de cooperación interétnicas para el manejo integrado de los recursos naturales. De esta manera, la integración de las economías familiares y comunales permitieron el intercambio y consumo de productos dentro de amplios territorios étnicos (mayas, incas, etc.). Al mismo tiempo, se establecieron reglas sociales para la regulación colectiva de la producción, a través de largos procesos de observación de la naturaleza, experimentación productiva, innovación técnica e intercambio de saberes. En este sentido, las identidades étnicas fueron significando y definiendo diferentes sistemas de recursos naturales.

Es en este sentido que la cultura está integrada dentro de las condiciones generales de una producción sustentable; las identidades étnicas y los valores culturales, así como las prácticas comunales para el manejo colectivo de la naturaleza, fueron y son la base para el desarrollo del potencial ambiental para el desarrollo sustentable de cada región y cada comunidad. La cultura se convierte así en un principio activo para el desarrollo de las fuerzas productivas en un paradigma alternativo de sustentabilidad; la productividad ecológica y la innovación tecnológica están entretejidos con procesos culturales que definen la productividad social de cada comunidad; y éstas a su vez se enlazan para generar una economía global alternativa, fundada en las fuerzas productivas de la naturaleza y en los sentidos creativos de la cultura (Leff, 1993,1994a).

En las culturas tradicionales, el conocimiento, los saberes y las costumbres están entretejidas en cosmovisiones, formaciones simbólicas y sistemas taxonómicos a través de las cuales clasifican a la naturaleza y ordenan los usos de sus recursos; la cultura asigna de esta manera valores-significado a la naturaleza, a través de sus formas de cognición, de sus modos de nominación y de sus estrategias de apropiación de los recursos. La cultura se inscribe en las múltiples funciones de la naturaleza a través de las prácticas agrícolas, de preservación de los procesos ecológicos, de protección de la erosión y mantenimiento de la fertilidad del suelo; de conservación de la diversidad genética y biológica; de regeneración selectiva de especies útiles; de manejo integrado de recursos naturales silvestres y especies cultivadas; y de la innovación de sistemas agroecológicos altamente productivos, como los camellones peruanos y las chinampas mexicanas.

Diferentes estudios sobre los estilos étnicos de apropiación del medio ambiente en distintas regiones del mundo, han mostrado sus detallados conocimientos de los suelos, lo que les permite usarlos eficientemente, obteniendo beneficios sustentables dentro de los límites de las condiciones ambientales y socioeconómicas, mientras se preserva la base de sus recursos. Estas prácticas indígenas están siendo reproducidas en diferentes lugares como estrategias de supervivencia cultural y desarrollo sustentable (Parra, 1993).

En las áreas rurales del tercer mundo, las prácticas sociales y productivas están íntimamente asociadas a valores y procesos simbólicos que organizan formaciones culturales y permiten la apropiación social y la transformación del medio ambiente. La organización cultural regula el uso de recursos para satisfacer las necesidades de sus miembros. A través de procesos simbólicos se configuran mecanismos culturales que norman el acceso social a la naturaleza, dan forma al desarrollo 
tecnológico y regulan los ritmos de extracción y transformación de los recursos. Las relaciones de parentesco, las formas de reciprocidad, las formas de propiedad comunal y los derechos territoriales favorecen el uso sustentable de los recursos naturales. El acceso socialmente sancionado a la naturaleza se define a través de tradiciones culturales que establecen las formas de tenencia de la tierra, de propiedad y manejo de recursos ambientales, las relaciones de género y edad, la división del trabajo y la distribución de actividades productivas. Estas condiciones sociales y racionalidades culturales especifican los patrones de uso de los recursos naturales de las culturas andinas, mesoamericanas y amazónicas del continente.

Un ejemplo ilustrativo en el mundo lo constituyen las culturas tradicionales en la India han establecido relaciones específicas con el medio ambiente, desarrollando prácticas de conservación a través de la diversificación y complementación de nichos ecológicos ocupados por los grupos endogámicos de diferentes regiones. Cada familia, tribu o casta social tiene derechos específicos para explotar un área determinada de tierra o cierto recurso natural bajo regulación comunal (Gagdil y Iyer, 1993). Estas prácticas incluyen restricciones sobre el territorio que puede ocupar un grupo social y extraer recursos, con técnicas, métodos y períodos autorizados para la explotación de bosques y animales, estableciendo una especialización ecológica por cada casta en la apropiación y uso de la naturaleza (Gagdil, 1985).

Estas prácticas de uso integrado y múltiple de recursos naturales están basadas en normas culturales y conocimientos tradicionales que codifican el ambiente, preservando la biodiversidad e incrementando la capacidad de las comunidades para satisfacer sus necesidades materiales y sus aspiraciones culturales (Toledo y Argueta, 1993). La naturaleza es al mismo tiempo un recurso económico y un patrimonio cultural. Estas estrategias culturales para el manejo productivo de la naturaleza ofrecen principios para incrementar la productividad sustentable de los recursos naturales, preservando las condiciones para el desarrollo de racionalidades alternativas y prácticas diversificadas de construcción y manejo de la naturaleza, y de coevolución con la cultura.

La percepción holística del medio ambiente juega un papel fundamental en los sistemas cognitivos de las sociedades tradicionales. Su visión del mundo integra sus mitos y rituales a sus prácticas productivas; su conocimiento de los fenómenos geofísicos (fases lunares y estaciones; ciclos bio-geo-químicos, ecológicos y ciclos hidrológicos) está asociado al conocimiento de diferentes tipos de suelos y condiciones topográficas, permitiendo un aprovechamiento complementario del espacio ecológico y generando estrategias de uso múltiple e integrado de los recursos.

Los valores culturales, entretejidos en el conocimiento, en las formaciones ideológicas y en la organización social y productiva, constituyen así condiciones para el desarrollo sustentable. Estas formas de cohesión social y autosuficiencia permiten hoy la supervivencia de diferentes poblaciones. A su vez, estas prácticas de uso múltiple de los recursos naturales están reafirmando identidades y enraizando una racionalidad ambiental en territorios culturales para el desarrollo sustentable.

\section{La localización de la globalización}

Hoy, la globalización está alcanzando los límites del logocentrismo, del centralismo económico y de la concentración del poder. Una fuerza centrífuga está siendo generada hacia la descentralización económica, la autonomía de las comunidades y la apertura del conocimiento, de donde emergen nuevos significados que reorientan el curso de la historia y de la civilización humana. Aun cuando el discurso del desarrollo sustentable está siendo asimilado por la racionalidad económica y por las políticas de capitalización de la naturaleza, los principios de la sustentabilidad se están arraigando en el ámbito local a través de la construcción de nuevas racionalidades productivas, sustentadas en valores y significados culturales, en las potencialidades ecológicas de la naturaleza, y en la apropiación social de la ciencia y tecnología.

Mientras la globalización promueve la distribución espacial de su lógica autocentrada, penetrando cada 
territorio, cada ecosistema, cada cultura y cada individuo, las políticas de la localidad están construyendo una globalidad alternativa desde la especificidad de los ecosistemas, la diversidad cultural y la autonomía de las poblaciones locales, sustentadas en una racionalidad ambiental.

Para construir esta racionalidad ambiental es necesario desenterrar las condiciones ecológicas de sustentabilidad y descongelar el tiempo en el que han quedado adormecidos los sentidos culturales, negados y desconocidos por el egocentrismo y la megalomanía de la racionalidad económica. Esta defensa de medio ambiente local no solo depende de la valoración económica de la naturaleza y de la cultura, sino de la actualización y reconfiguración de las identidades y la emergencia de nuevos actores sociales que puedan construir una nueva racionalidad productiva, basada en los potenciales ecológicos de la naturaleza y en los significados culturales de los pueblos.

Esto abre la pregunta sobre la relación entre significado y conocimiento. Los seres humanos indagan la realidad y van a la conquista del mundo investidos de cosmovisiones e ideologías; observan con lentes conceptuales y armaduras paradigmáticas. El saber ambiental no está construido por la objetividad de la naturaleza aprehendida a través de un orden económico preconcebido. Es sobre todo la reconstrucción del conocimiento desde nuevas visiones, potencialidades y valores, guiados por nuevas significaciones y sentidos civilizatorios (Leff, 1998).

El conocimiento local no es solamente el arsenal de técnicas y saberes construidos por la práctica. El conocimiento local no suma las condiciones empíricas a estudios abstractos. No es la sumisión de particularidades locales a racionalidades universales dominadoras y hegemónicas. El conocimiento local está construido por significados elaborados a través de procesos simbólicos que configuran estilos étnicos de apropiación del mundo y de la naturaleza. Esto conduce a desentrañar los sentidos del tiempo en la forja, sedimentación y actualización de las identidades y formaciones culturales —de las diversas formas culturales del ser-enraizadas en significados y paisajes, en diferentes épocas y lugares, de sus flujos a través del curso de la historia pasada y de su irrupción repentina e inesperada al fin del siglo $\mathrm{XX}$, con la revalorización del etnicismo y la reivindicación de la autonomía de los pueblos indios.

Así, el conocimiento pasado, dominado y oprimido, es reconstruido por un nuevo saber local y personal, hibridizado con los nuevos discursos de la globalización y de la sustentabilidad, con la ciencia y la tecnología modernas, abriendo sendas inéditas en la historia. La sustentabilidad es una cuestión del ser y del tiempo, y no de la economización de la naturaleza. El devenir no es la evolución de una esencia genética y de racionalidades preestablecidas, sino la actualización de identidades que buscan vencer las formas de poder que han sujetado sentidos civilizatorios alternativos, para redescubrir los mundos ocultados, las prácticas sepultadas y los saberes subyugados, para reconducir la historia por los cauces de la diversidad cultural.

La globalización guiada por una racionalidad tecnológica unidimensional y por la racionalidad económica orientada hacia la maximización del beneficio económico en el corto plazo, ha resultado en un planeta contaminado, en un mundo saturado y en una economía insustentable. Desde los límites de esta racionalidad de la modernidad, la historia se está reabriendo a un futuro sustentable, fertilizado por los flujos divergentes del ser y del tiempo. La emergencia de la complejidad, la diferencia y la autonomía están confrontando las concepciones ontológicas y epistemológicas de la civilización occidental desde el pensamiento metafísico de la Antigua Grecia, hasta el "iluminismo" científico que inaugura la modernidad, llevando a la tecnologización del mundo y a la capitalización de la naturaleza que marcan la era actual de globalización hegemónica.

El renacimiento de las etnias y la revalorización de la diversidad cultural está generando una nueva comprensión de las relaciones de lo uno y lo otro, de lo objetivo y lo subjetivo, de lo real y lo simbólico, de la cultura y la naturaleza. Este crisol cultural, donde se funden lo ideal y lo material (Godelier, 1984), ha construido diversos mundos de vida, a través de significados diferenciados y del posicionamiento del ser en diversos ambientes.

Las sociedades tradicionales y las economías 
locales no producen solo valores de uso y de cambio; también producen "significados de uso" que reflejan la compleja relación del orden simbólico-natural en las relaciones de producción económico-políticas. La naturaleza no está solamente codificada por un lenguaje y guiada por una racionalidad económica dominante. Las invenciones de la humanidad, grabadas en la memoria colectiva de los pueblos, ya no sólo son interpretadas por los antropólogos; hoy reemergen como un proceso de resignificación, reafirmación y actualización de las identidades de los pueblos, como una "relocalización" de sus mundos de vida. "Los entes culturales" pasados no sólo son recordados sino recodificados, recobrando aquello que alguna vez fue depositado en la memoria de cultura, desenredando la madeja del tiempo y forjando una nuevo vínculo entre el pasado y el futuro.

El tiempo es dinero en un mundo economizado. El significado forja al tiempo en la realidad simbólica de la cultura y la historia. El tiempo no es una sucesión de eventos. La densidad del destino y la elasticidad del tiempo simbólico en la consciencia humana y sus acciones sociales, provienen de significados enraizados en identidades culturales diversas que se transfieren a través de generaciones, generando una genealogía de racionalidades diferenciadas. La cuestión de espacio y lugar no es solamente la de un sincretismo cultural y un tiempo sincrónico, sino la de una diacronía marcada por la supervivencia enigmática y la actualización de culturas tradicionales; de su resistencia y asimilación con otras culturas; de su encuentro, hibridación y demarcación hoy frente al discurso y las políticas de la globalización.

El logocentrismo de la racionalidad científica sobre la que está suspendida la globalización, se dirige hacia la búsqueda de una base pragmática, sustantiva y empírica de las acciones para asegurarse el transito hacia la sustentabilidad. Sin embargo, los procesos ecológicos y los valores culturales son inconmensurables con la racionalidad económica. El mercado es incapaz de asignar valores reales a los servicios ecológicos y a las condiciones comunales para la sustentabilidad debido a su estructura teórica mecanicista, a la imposibilidad de reducir futuros sustentables y las diversas y heterogéneas condiciones de sustentabilidad a los valores unitarios del mercado.
La ecología, propuesta como la ciencia por excelencia de las interrelaciones biofísicas, de hecho es un paradigma no espacial, una lógica que carece de referencias geo-gráficas para concretar el espacio y el lugar. De tal manera, Tricart $(1978,1982)$ advirtió la necesidad de integrar la ecología con una geografía más descriptiva en un paradigma interdisciplinario eco-geográfico. La emergencia de la geo-referencialidad es la necesidad de enraizar la sustentabilidad en condiciones locales, pero que ignora que estos referentes provienen de la significación que imprime la cultura en la naturaleza.

El espacio y el lugar están siendo "reinventados" desde las identidades culturales para enraizar y especificar las condiciones de sustentabilidad, allí donde las construcciones del logocentrismo de las ciencias se han extraviado, perdiendo sus referentes geográficos y sus sentidos culturales. Sin embargo, no sólo se trata de la necesidad de dar soporte empírico y referencias locales a una racionalidad globalizadora y globalizante, de encontrar el balance y la simetría entre lo global y lo local, el punto de equilibrio entre conservación y crecimiento económico. Los procesos donde arraiga la sustentabilidad no son sólo de carácter "material-objetivo", sino "simbólico-subjetivo", marcados por principios, valores y significados diferenciados.

Desde la consciencia de la finitud de la existencia, damos significado a los eventos de nuestras vidas; en forma similar, la muerte de la naturaleza y la cultura ha reactivado sus historias diferenciadas. La construcción de una racionalidad ambiental entraña el resurgimiento y resignificación de la naturaleza y de la cultura para liberarse de la opresión que sobre el saber y la acción ejercen la economización y la tecnologización del mundo. El renacimiento de las identidades es la precipitación de los tiempos contenidos por una historia de homogeneización y dominación de la diversidad y de la diferencia.

Desde la perspectiva de una política del espacio, del lugar y del tiempo, la autonomía de la gente no puede ser concebida como el "empoderamiento desde arriba" de los oprimidos. La autonomía cultural no puede ser graciosamente otorgada a quienes han quedado marginados y excluidos de las razones que han organizado y legitimado al mundo actual, aún en esta era de 
democracia, ciudadanía y derechos humanos. El derecho a la autonomía es el reclamo de las "localidades" oprimidas: culturas locales, conocimiento local, gente local. Estas demandas sociales surgen de la actualización de las identidades, la hibridación en tiempo y espacio de diferentes formas del ser. La resistencia a la capitalización de la naturaleza es la expresión de la resiliencia cultural que reacciona para preservar sus identidades frente a las perturbaciones externas. Es a través de la reconstrucción del ser que la autonomía puede dirigirse hacia la autogestión de las condiciones de vida de la gente.

\section{La política del lugar y del espacio, el derecho a la identidad y la cuestión del ser y el tiempo}

La política del lugar y el espacio está emergiendo como un reclamo social, movilizada por los nuevos derechos a la identidad cultural de los pueblos (CNDH, 1999; Sandoval y García, 1999), legitimando reglas más plurales y democráticas de convivencia social. La reafirmación de la identidad es también la manifestación de lo real y de lo verdadero frente a la racionalidad económica que se ha constituido como el más alto grado de racionalidad del ser humano, ignorando a la naturaleza y a la cultura, generando la hiperrealidad entrópica insustentable que gobierna nuestra existencia.

El lugar es el territorio donde la sustentabilidad se enraíza en bases ecológicas e identidades culturales. Es el espacio social donde los actores sociales ejercen su poder para controlar la degradación ambiental y para movilizar potenciales ambientales -la sinergia generada por procesos sincrónicos y diacrónicos - en proyectos autogestionarios generados para satisfacer necesidades, aspiraciones y deseos de la gente, que la globalización no puede cumplir.

El lugar es el locus de las demandas y los reclamos de la gente por la degradación ambiental, así como sus capacidades de reconstruir sus mundos de vida. El nivel local es donde se forjan las identidades culturales, donde se expresan como una valorización social de los recursos económicos y como estrategias para la reapropiación de la naturaleza. Si la globalización es el espacio donde las sinergias negativas hacen manifiestos los límites del crecimiento, lo local es el espacio donde emergen las sinergias positivas de la racionalidad ambiental.

El espacio es el punto de encuentro de procesos sincrónicos y diacrónicos, donde se precipitan tiempos y momentos diferenciados. Lo local es el lugar en donde se articulan identidades culturales y potencialidades ecológicas. Es pues el lugar de convergencia de los tiempos de la sustentabilidad: los procesos de restauración y productividad ecológica; de innovación y asimilación tecnológica; de reconstrucción de identidades culturales.

Siguiendo a Evans Pritchard, el tiempo se estructura alrededor de eventos significativos, tanto sociales como económicos. De esta manera, cada cultura, define sus "temporalidades" a través de su cosmología y sus sistemas simbólicos (Pitt, 1985). El tiempo no es solo la medida de eventos externos (fenómenos geofísicos, ciclos ecológicos, procesos de degradación y regeneración de la naturaleza), sino el flujo de eventos internos significativos, la permanencia de "presencias" a través de la historia, la actualización de las identidades étnicas y "seres culturales". El tiempo libera a la naturaleza de-signada y fijada a visiones predeterminadas, abriendo los cauces para la construcción de una naturaleza resignificada por los sentidos de la identidad y la cultura.

Una nueva política del espacio, del lugar y el ser está siendo construida a partir del sentido del tiempo en las luchas actuales por la identidad, por la autonomía y por el territorio. Es una política del ser lo que subyace al clamor por el reconocimiento de los derechos a la supervivencia, a la diversidad cultural y la calidad de vida de los pueblos; es una política del devenir y la transformación, que valoriza el significado de la utopía como el derecho de cada individuo y cada comunidad para forjar su propio futuro. Los territorios culturales están siendo fertilizados por un tiempo pleno de significados. No es sólo la reivindicación de los derechos culturales que incluyen la preservación de los usos y costumbres de sus lenguas autóctonas y sus prácticas tradicionales, sino una política cultural para la reconstrucción de identidades y significados existenciales, para proyectar sus seres colectivos trascendiendo un futuro prefijado y excluyente; es resistencia a la hegemonía homogeneizante y afirmación 
de la diversificación creativa de la vida, construida desde la heterogénesis cultural-ecológica.

Las identidades nunca son idénticas. Al contrario, el clamor por la igualdad y la sustentabilidad desde esta política cultural por la identidad es una lucha por la diversidad, por el derecho de ser diferente. Es el derecho a la singularidad y a la autonomía frente al forzamiento de la universalidad impuesta por una globalización dominadora.

Esta política del ser y el devenir, está emergiendo en la reconstitución de las identidades y la innovación de proyectos culturales en el tránsito a la democracia y a la sustentabilidad. La construcción de una nueva racionalidad ambiental se está forjando en movimientos emergentes de los pueblos indígenas. Esta es una política que "enfatiza el devenir más que el ser; que implica un posicionamiento más que un esencialismo, la discontinuidad tanto como la continuidad". (Escobar, 1997a:18).

Esta política cultural esta siendo forjada por movimientos emergentes de las comunidades indígenas, como el movimiento negro reciente en la Costa del Pacífico en Colombia (Grueso, Rosero y Escobar, 1997):

Los principios primordiales del movimiento para la organización política [son] el derecho a una identidad, esto es, el derecho a ser negro de acuerdo a la lógica cultural y la visión del mundo arraigada en la experiencia negra [...] para la reconstrucción de la propia consciencia negra y el rechazo del discurso dominante de la "igualdad" con su obliteración concomitante de la diferencia. Segundo, el derecho a un territorio como un espacio para ser y como elemento esencial para el desarrollo de la cultura. Tercero, el derecho a una autonomía política como un prerequisito para la práctica de ser, con la posibilidad de promover la autonomía social y económica. Cuarto, el derecho de construir su propia visión del futuro, del desarrollo y de sus prácticas sociales basadas en los usos y costumbres en la producción y la organización social. Quinto, el principio de solidaridad con las luchas de las poblaciones negras del mundo en busca de visiones alternativas [...] Los activistas conceptualizan el territorio como un espacio para la creación de futuros, para la esperanza y la continuación de la existencia." (Escobar, 1997a: 14-16)
De esta manera, las poblaciones indígenas están generando nuevos derechos culturales para recuperar el control sobre su territorio como un espacio ecológico, productivo y cultural para reapropiarse un patrimonio de recursos naturales y significados culturales. La racionalidad ambiental está siendo internalizada por nuevos actores sociales, expresándose como una demanda política que guía nuevos principios para la valorarización del ambiente y para la reapropiación de la naturaleza, y arraigándose en nuevos territorios y nuevas identidades (Leff, 1995).

La política cultural está emergiendo junto con la construcción de un conocimiento del ambiente donde el tiempo-significante habita el ser (Leff, 2000). La política cultural se está forjando en el crisol de la diferencia y la diversidad, de actores sociales que incorporan diferentes significados y prácticas culturales, en la hibridación de diferentes procesos materiales y simbólicos, en la actualización de seres hechos de tiempo, de vida y de historia. Estamos atestiguando la "conjunción de diferentes regímenes históricos y epistémicos”, el despertar de tradiciones y la supervivencia de significados. Para entender esta resiliencia cultural como expresión del tiempo en las identidades, tendremos que "dilucidar la genealogía de sus diferentes racionalidades" (Escobar) y comprender su articulación en la forja de la racionalidad ambiental.

\section{El tiempo, la identidad y la forja de la racionalidad ambiental.}

La historia está hecha de tiempo; es la emergencia y la expresión de tiempos inmemorables, de tiempos congelados. La historia guarda sus innovaciones en secreto. Así, los 60's fueron el tiempo de la irrupción del pensamiento de la complejidad y de la democracia, al tiempo que la crisis ecológica y la explosión demográfica marcaron los límites del crecimiento. 1989 fue la caída del socialismo real; 1992 fue la emergencia de la sustentabilidad en la saturación racionalidad económica global y el despertar de una nueva conciencia de los pueblos indios americanos luego de 500 años de con- 
quista y explotación. La irrupción en la historia de estos eventos inéditos es expresión de fuerzas internas del ser que están rompiendo las cadenas de las estructuras dominantes de poder.

El presente es el cruce de tiempos heterogéneos, desde la explosión y actualización de tradiciones congelados y bloqueadas por los poderes dominantes, hasta el entrelazamiento de diferentes racionalidades que han forjado diversas formas de relaciones entre la naturaleza y la cultura. Es la supervivencia del pasado en el presente, la configuración de nuevas identidades y la construcción de nuevas utopías que reorientan al mundo hacia alternativas futuras.

Hoy, la historia se forja por la re-emergencia del ser. Historias acallada, que parecían haber perdido su memoria y sus tradiciones, se están resignificando por nuevos propósitos, orientando una política del ser y del tiempo, y reconstruyendo eventos históricos pasados a través de la actualización de significados oprimidos.

Heidegger se preguntó así por

Esta quietud del acontecer [que] no es ausencia de la historia, sino una forma básica de su presencia. Lo que conocemos generalmente como pasado y lo que nos representamos en primer término como tal, es casi siempre sólo la 'actualidad' de un momento pasado, [...] lo que pertenece siempre a la historia pero no es propiamente historia. El mero pasado no agota lo sido. Este está presente todavía, y su forma de ser es una peculiar quietud del acontecer, cuya forma se determina a partir de aquello que acontece. La quietud es sólo un movimiento que se detiene en si, y es con frecuencia más inquietante que éste (Heidegger, 1975, p. 44-45).

Esta "quietud" está adquiriendo nuevas voces, forjando nuevas identidades, movilizando nuevos actores, fertilizando nuevos seres, abriendo nuevos horizontes en la historia. Y esto es más y otra cosa que el reencuentro de diversas historias, la hibridación de procesos ontológicos diferenciados, los círculos viciosos de procesos de crecimiento que se acumulan y desbordan en catástrofes ecológicas, o la confluencia de procesos sinergéticos para construir una productividad ecotecnológica sustentable (Leff, 1994a); es más que un proceso evolucionario y un cambio revolucionario trascendental en la historia. Mas allá de las modificaciones transgénicas de la vida y de la economización de la naturaleza, las nuevas identidades están forjando una mutación histórica y la emergencia de nuevos tiempos.

Estas nuevas identidades no son la reencarnación y la actualización de seres de antaño en tiempos, lugares y espacios "modernos". Las nuevas identidades se están configurando en un proceso de emancipación en el contexto de realidades presentes, en resistencia y confrontación con el orden global dominante que tiende a asimilar toda diferencia en su hegemonía homogénea (Laclau, 1996). Las identidades híbridas emergentes se están forjando en una lucha política -en campos antagónicos- por la apropiación de la naturaleza y la resignificación de mundos de vida. Estas luchas van más allá del derecho al multiculturalismo, a la expresión de diferencias en un orden global finalizado, a un sistema político consensuado en torno a una razón globalizante; trascienden la lógica de la democracia representativa, abriéndose a la expresión de la diferencia, la otredad y la alternativa, ante el ensimismamiento y el cierre del régimen global imperante.

En esta era del vacío (Lipovetsky, 1986), en un tiempo en el que el mundo ha quedado desustantivado y desreferenciado, ante la evaporación y disolución de los sentidos de la vida y los propósitos históricos construidos a través de ideologías políticas, normas jurídicas y morales sociales, fundadas en la construcción metafísica, epistemológica y ética de la civilización occidental, las identidades aparecen como el lugar del ser donde convergen los procesos ontológicos y se plasman los sentidos existenciales de lo humano.

El lugar de la identidad no podrá ser un sitio del ser. Los derechos comunales se nutren de sentido y de diversidad; no se plasman en un "lugar común" o en un "futuro común". El hombre, por su voluntad de saber y su poder de apropiación de la naturaleza ha transformado al mundo, cambiando leyes, desviando inercias, generando significados, forjando sentidos. La complejidad ambiental se está configurando en el tránsito hacia el tercer milenio, desde la modernidad truncada hacia una posmodernidad indefinida, para construir una 
nueva racionalidad, capaz de resignificar y reabrir los cauces de la historia (Leff, 1994b, 2000). La diversidad y la diferencia se convierten en principios que trascienden a la negatividad dialéctica de oposiciones y antagonismos dentro del sistema de poder establecido. La racionalidad ambiental abre la lógica de la unidad hacia el sentido de la identidad como un sistema de diferencias donde sea posible el reconocimiento del otro, la alteridad y el advenimiento de "lo que aún no es" (Levinas, 1977). En este sentido se están constituyendo las nuevas identidades y racionalidades como fuente y soporte de nuevos saberes, nuevos territorios y nuevos seres.

El tiempo que forja un futuro sustentable no es sólo aquella dimensión del tiempo inherente a eventos y procesos externos y objetivos, sino un tiempo

\section{BIBLIOGRAFÍA}

ALCORN, J. Process as Resource: The Traditional Agricultural Ideology of Bora and Huastec Resource Managment and its Implications for Research1. Advances in Economic Botany, n. 7, p. 63-77, 1989.

ALTIERI, M.; C. NICHOLLS. Agroecología: teoría y práctica para una agricultura sustentable. Serie textos básicos para la formación ambiental, México. n. 4, PNUMA/ORPALC, 2000.

BOEGE, E. Los maztecos ante la nación. México: Siglo XXIEds, 1988.

BURGOA, M.; GALINDO, A. La utopía andina. Allpanchis, v. 20, 1982.

CEPAL/PNUMA. Sobrevivencia campesina en ecosistemas de altura, 2 v. Santiago de Chile. 1983.

CNDH. El Derecho a la identidad culturtal. Gaceta, México. n. 103. Comisión Nacional de Derechos Humanos.

CONDARCO, R.; J. MURRA. La teoría de la complementariedad vertical-ecosimbótica. La Paz: Hisbol, 1987.

DE LA TORRE, C.; M. BURGOS (Comps.). Andenes y fenomenológico: el tiempo que construye la historia, el tiempo que da forma a diferentes mundos de vida, el tiempo que toma cuerpo en identidades que configuran sentidos existenciales, que movilizan procesos sociales y emancipan voluntades de cambio; el tiempo que desencadena al mundo con nuevos significados que organizan lo simbólico y lo material en el encuentro de la ecología y la cultura.

El proyecto civilizador que pretende unificar al mundo está muerto: el Dios único, la idea absoluta, la unidad de la ciencia y la globalización del mercado. La historia se abre a una diversidad de modos de vida y racionalidades productivas, marcadas por los límites y potencialidades de la naturaleza y por los sentidos del tiempo.

camellones en el Perú andino. Lima: CONCYTEC, 1986.

DENEVAN, W. Tipología de configuraciones agrícolas prehispánicas. América Indígena, n. 40, p. 610-152, 1980a.

DENEVAN, W. El asentamiento nativo de los llanos de moxos: una sabana inundada periódicamente en el noreste de Bolivia, 1980b.

DESCOLA, Ph. La selva culta. Simbolismo y praxis en la Ecología de los Achuar. Quito: Ediciones Abya-Yala. 1996.

ESCOBAR, A. Cultural politics an biological diversity: state, capital and social movements in the pacific coast of Colombia. In: FOX, R.; STARN, O. (Eds.), Between Resistance ans Revolution. New Brunswick, 1997a.

ESCOBAR, A. The Place of Nature and the Nature of Place: Local Knowledge and Alternative Worlds, Simposium on Ethnobotanics and Epistemology, II International Congress on Ethnobotanics, Merida. Yucatan, Mexico, October 12-17, 1997.

FLORES GALINDO, A. 1988. Buscando un inca. Identidad y utopía en los Andes, Lima. In: MARTÍNEZ-ALIER, J. Ecology and the poor: A neglected dimension of Latin 
LEFF, E. Espacio, lugar y tiempo: la reapropiación social de la natureza...

america history. Journal of Latin American Studies. n. 23, p. 621-639.

GAGDIL, M. Social Restrainrs on Resource Utilization: The Indian Experience. In: McNEELY, J.; PITT, D. Culture and Conservation, 1985.

GAGDIL, M.; P. IYER . La diversificación en el uso de los recursos de propiedad común en la sociedad india. In: LEFF; CARABIAS 1993, p. 551-573.

GLIGO, N.; MORELLO, J. Notas sobre la Historia Ecológica de América Latina. In: O. SUNKEL; N. GLIGO. Estilos de desarrollo y Medio Ambiente en la América Latina. México: FCE. 1980.

GODELIER, M. L'idéel et le matériel. Paris: Fayard.

GRUESO, L.; C. ROSERO; A. Escobar. The process of black community organizing in the southern pacific coast region of Colombia. 1997. Mimeo.

GRÜNBERG, G. (Coord). Articulación de la diversidad. Pluralidad etnica, autonomías y democratización en América Latina. Quito: Ediciones Abya-Yala. 1995.

HEIDEGGER, M. La pregunta por la cosa. Buenos Aires: Ed. Alfa, 1975.

LACLAU,E. Emancipations. London/New York: Verso. 1996.

LEFF, E. Ecología y capital. Racionalidad ambiental, democracia participativa y desarrollo sustentable. México: Siglo XXI Editores/IIS-UNAM, 1994a.

LEFF, E. Sociología y ambiente: formación socioeconómica, racionalidad ambiental y transformaciones del conocimiento. In: LEFF, E. (Coord.). Ciencias Sociales y formación ambiental. Barcelona: GEDISA/CIICH-UNAM/ PNUMA, 1994b.

LEFF, E. De Quién es la naturaleza? Sobre la reapropiación social de los recursos naturales. Gaceta Ecológica, n. 37, México: INE-SEMARNAP, p. 58-64, 1995.

LEFF, E. Saber Ambiental: sustentabilidad, racionalidad, complejidad. Poder. México: Siglo XXI/UNAM/PNUMA, 1998.

LEFF, E. Pensar la complejidad ambiental. In: LEFF, E. (Coord.). La complejidad ambiental. Colección aprender a aprender. Siglo XXI/ CIICH-UNAM/PNUMA. 2000
LEFF, E., J. CARABIAS; A. I. BATIS. Recursos naturales, técnica y cultura. Estudios y experiencias para un desarrollo alternativo. Centro de investigaciones interdisciplinarias en humanidades. Serie Seminarios, $n$. 1, México: UNAM. 1990.

LEFF, E.; J. CARABIAS. Cultura y manejo sustentable de los recursos naturales. México: CIICH-UNAM/ Miguel A. Porrua Eds. 1993.

LEVINAS, E. Totalidad e infinito. Ensayo sobre la Exterioridad. Salamanca: Ediciones Sígueme. 1997.

LÉVI-STRAUSS, C. Structuralism and Ecology. Social Science Information, v. 12, n. 1, p. 7-23, 1972.

LIPOVETSKY, G. La era del vacío. Barcelona: Editorial Anagrama. 1986.

MASUDA, S.; SHIMADA, I.; C. MORRIS (Eds.). Andean ecology and civilization: an interdisciplinary persective on andean ecological complementary. Tokyo, 1985.

MORELLO, J. Conceptos para un manejo integrado de los recursos naturales. In: LEFF, E. Los problemas del conocimiento y la perspectiva ambiental del desarrollo. México: Siglo XXI, 1986.

MORELLO, J. Insumos para la agenda ambiental LatinoAmericana. In: BID/PNUD. Nuestra propia agenda. Washington, D.C, 1990.

MURRA, J. V. Formaciones económicas y políticas del Mundo Andino. Perú: IEP, 1975.

PARRA, M. La producción silvoagropecuaria de los indígenas de los altos de Chiapas. In: LEFF, E. Y; J. CARABIAS, v. 2, p. 445-487, 1993.

PITT, D. 1985. Towards Ethnoconservation. In: McNEELY J.; D. PITT. Culture and conservation: the human dimension in enviromental planning. IUCN, Croom Helm.

ROMANINI, C. Ecotécnicas para el trópico húmedo. CECODES-CONAC; T/PNUMA, 1976.

SANDOVAL, I. E.; G. García Colorado. El derecho a la identidad cultural. Instituto de Investigaciones Legislativas. México: H. Cámara de Diputados, 1999.

SAN MARTÍN ARZABE, G. H. Las formas tradicionales de organización social y la actividad en el medio indíge- 
LEFF, E. Espacio, lugar y tiempo: la reapropiación social de la natureza...

na: las sabanas de moxos. In: E. LEFF, CARABIAS; A. I. BATIS. Recursos naturales, técnica y cultura, 1990.

TOLEDO, V.M. Ecología del modocampesino de producción. Antropología y Marxismo, México. n. 3, p. 35-55, 1980.

TOLEDO, V. M.; A. ARGUETA. Naturaleza, producción y cultura en una región indígena de México: las lecciones de Pátzcuaro. In: LEFF; CARABIAS. 1993, p. 413-443.

TRICART, J. Vocation des terres, ressources ou contraintes et developpement rural. Herodote, v. 12, p. 66-75, 1982.
TRICART, J. Geographie/Ecologie. Herodote, n. 26, 1988.

URIBE, R. Medio Ambiente y comunidades indígenas del Sureste. Prácticas tradicionales de producción, rituales y manejo de recursos. México: UNESCO/Gobierno de Tabasco, Villahermosa, 1988.

VARÈSE, S.; G. MARTIN. Ecología y producción en dos áreas indígenas de México y Perú: experiencias y propuestas para un desarrollo culturalmente sustentable. In: LEFF; CARABIAS. v. 2, p. 717-740. 1993. 